\title{
Parallel Working-Set Search Structures
}

\author{
Kunal Agrawal \\ Washington University in St. Louis
}

\author{
Seth Gilbert \\ National University of Singapore
}

\author{
Wei Quan Lim \\ National University of Singapore
}

\begin{abstract}
In this paper we present two versions of a parallel working-set map on $p$ processors that supports searches, insertions and deletions. In both versions, the total work of all operations when the map has size at least $p$ is bounded by the working-set bound, i.e., the cost of an item depends on how recently it was accessed (for some linearization): accessing an item in the map with recency $r$ takes $O(1+\log r)$ work. In the simpler version each map operation has $O\left((\log p)^{2}+\log n\right)$ span (where $n$ is the maximum size of the map). In the pipelined version each map operation on an item with recency $r$ has $O\left((\log p)^{2}+\log r\right)$ span. (Operations in parallel may have overlapping span; span is additive only for operations in sequence.)

Both data structures are designed to be used by a dynamic multithreading parallel program that at each step executes a unit-time instruction or makes a data structure call. To achieve the stated bounds, the pipelined version requires a weak-priority scheduler, which supports a limited form of 2-level prioritization At the end we explain how the results translate to practical implementations using work-stealing schedulers.

To the best of our knowledge, this is the first parallel implementation of a self-adjusting search structure where the cost of an operation adapts to the access sequence. A corollary of the working-set bound is that it achieves work static optimality: the total work is bounded by the access costs in an optimal static search tree.
\end{abstract}

The full version of this paper is at http://arxiv.org/abs/1805.05787.

\section{ACM Reference Format:}

Kunal Agrawal, Seth Gilbert, and Wei Quan Lim. 2018. Parallel Working-Set Search Structures. In SPAA '18: 30th ACM Symposium on Parallelism in Algorithms and Architectures, July 16-18, 2018, Vienna, Austria. ACM, New York, NY, USA, 12 pages. https://doi.org/10.1145/3210377.3210390

\section{Introduction}

Map (or dictionary) data structures, such as binary search trees and hash tables, support inserts, deletes and searches/updates (collectively referred to as accesses) and are one of the most used and studied data structures. In the comparison model, balanced binary search trees such as AVL trees and red-black trees provide a performance guarantee of $O(\log n)$ worst-case cost per access for a tree with $n$ items. Other kinds of balanced binary trees provide probabilistic or amortized performance guarantees, such as treaps and weight-balanced trees.

Permission to make digital or hard copies of all or part of this work for personal or classroom use is granted without fee provided that copies are not made or distributed for profit or commercial advantage and that copies bear this notice and the full citation on the first page. Copyrights for components of this work owned by others than the author(s) must be honored. Abstracting with credit is permitted. To copy otherwise, or republish, to post on servers or to redistribute to lists, requires prior specific permission and/or a fee. Request permissions from permissions@ acm.org.

SPAA '18, July 16-18, 2018, Vienna, Austria

(C) 2018 Copyright held by the owner/author(s). Publication rights licensed to ACM. ACM ISBN 978-1-4503-5799-9/18/07 . .\$15.00

https://doi.org/10.1145/3210377.3210390
Self-adjusting maps, such as splay trees [37], adapt their internal structure to the sequence of operations to achieve better performance bounds that depend on various properties of the access pattern (see [20] for a hierarchical classification). Many of these data structures make it cheaper to search for recently accessed items (temporal locality) or items near to previously accessed items (spatial locality). For instance, the working-set structure described by Iacono in [29] has the working-set property (which captures temporal locality); it takes $O(\log r+1)$ time per operation with access rank $r$ (Definition 1), so its total cost satisfies the working-set bound (Definition 2).

Definition 1 (Access Rank). Define the access rank of an operation in a sequence of operations on a map $M$ as follows. The access rank for a successful search for item $x$ is the number of distinct items in $M$ that have been searched for or inserted since the last prior operation on $x$ (including $x$ itself). The access rank for an insertion, deletion or unsuccessful search is always $n+1$, where $n$ is the current size of $M$.

Definition 2 (Working-Set Bound). Given any sequence $L$ of $N$ map operations, we shall use $W_{L}$ to denote the working-set bound for $L$, defined by $W_{L}=\sum_{i=1}^{N}\left(\log r_{i}+1\right)$ where $r_{i}$ is the access rank of the $i$-th operation in $L$ when $L$ is performed on an empty map.

\section{Parallel search structures}

Our goal in this paper is to design efficient self-adjusting search structures that can be used by parallel programs. Even designing a non-adjusting parallel or concurrent search structure is quite challenging, and there has been a lot of research on the topic.

There are basically two approaches. In the concurrent computing world, processes independently access the data structure using some variety of concurrency control (e.g., locks) to prevent conflicts. In the parallel computing world, data structures are designed to support executing operations in parallel, either individually or in batches.

For example, in the concurrent computing world, Ellen et al. [19] show how to design a non-blocking binary search tree, with later work generalizing this technique [13] and analyzing the amortized complexity [18]. However, these data structures do not maintain balance in the tree (i.e., the height can get large) and their cost depends on the number of concurrent operations.

An alternate approach (that bears some similarity to the implicit batching that we use) is software combining [22, 28, 32], where each processor inserts a request in a shared queue and a single processor sequentially executes all outstanding requests later. These works provide empirical efficiency but not worst-case bounds.

Another notable example is the CBTree [1,2], a concurrent splay tree that in real experiments achieves surprisingly good performance leading to an interesting hypothesis that self-adjustment may be even more valuable (in practice) in concurrent settings than sequential settings. However, the CBTree does not guarantee that it maintains the proper 'frequency balance', and hence does not provide the guarantees of a splay tree (despite much experimental success).

In the parallel computing world, there are several classic results in the PRAM model. Paul et al. [34] devised a parallel 2-3 tree such that $p$ synchronous processors can perform a batch of $p$ operations 
on a parallel 2-3 tree of size $n$ in $O(\log n+\log p)$ time. Blelloch et. al [8] show how pipelining can be used to increase parallelism of tree operations. Also, (batched) parallel priority queues [12, 15, 16, 36] have been utilized to give efficient parallel algorithms such as for shortest-path and minimum spanning tree [12, 16, 33].

More recently, in the dynamic multithreading model, there have been several elegant papers on parallel treaps [9] and how to parallelize a variety of different binary search trees [7] supporting unions and intersections, and also work on how to achieve batch parallel search trees with optimal work and span [4]. Other batch parallel search trees include red-black trees [23] and weight-balanced B-trees [21]. (We are unaware of any batched self-adjusting data structures.)

And yet, such concurrent/parallel map data structures can be difficult to use; the programmer cannot simply treat it as a black box and use atomic map operations on it from within an ordinary parallel program. Instead, she must carefully coordinate access to the map.

\section{Implicit batching}

Recently, Agrawal et al. [3] introduced the idea of implicit batching. Here, the programmer writes a parallel program that uses a black box data structure, treating calls to the data structure as basic operations. In addition, she provides a data structure that supports batched operations (e.g., search trees in [7, 9]). The runtime system automatically stitches these two components together, ensuring efficient running time by creating batches on the fly and scheduling them appropriately. This idea of implicit batching provides an elegant solution to the problem of parallel search trees.

\section{Our goals}

Our goal is to extend the idea of implicit batching to self-adjusting data structures - and more generally, to explore the feasibility of the implicit batching approach for a wider class of problems. In [3], they show how to apply the idea to uniform-cost data structures (where every operation has the same cost). ${ }^{1}$ In a self-adjusting structure, some operations are much cheaper than others, and additionally every operation may modify the data structure (unlike say AVL/redblack trees where searches have no effect on the structure), which makes parallelizing it much harder.

We present in this paper, to the best of our knowledge, the first parallel self-adjusting search structure that is distribution-sensitive with worst-case guarantees. In particular, we design two versions of a parallel map whose total work is essentially bounded by the Working-Set Bound for some linearization $L$ of the operations (that respects the dependencies between them).

\section{Parallel Programming Model}

The parallel data structures in this paper can be used in the scenario where a parallel program accesses data structures expressed through dynamic multithreading (see [14, Ch. 27]), which is the case in many parallel languages and libraries, such as Cilk dialects [24, 30], Intel TBB [35], Microsoft Task Parallel Library [38] and subsets of OpenMP [31]. The programmer expresses algorithmic parallelism through parallel programming primitives such as fork/join (also spawn/sync), parallel loops and synchronized methods, and does not provide any mapping from subcomputations to processors.

\footnotetext{
${ }^{1}$ They also provide some bounds for amortized data structures, where queries do not modify the data structure.
}

These types of programs are typically scheduled using a greedy scheduler [11, 27] or a nearly greedy scheduler such as workstealing scheduler (e.g., [10]) provided by the runtime system. A greedy scheduler guarantees that at each time step if there are $k$ available tasks then $\min (k, p)$ of them are completed.

We analyze our two data structures in the context of a greedy scheduler and a weak-priority scheduler (respectively). A weakpriority scheduler has two priority levels, and at each step at least half the processors greedily choose high-priority tasks and then lowpriority tasks - if there are at most $\frac{1}{2} p$ high-priority tasks, then all are executed. We discuss in Section 8 how to adapt these results for work-stealing schedulers.

\section{Main Results}

We present two parallel working-set maps that can be used with any parallel program $P$, whose actual execution is captured by a program DAG $D$ where each node is a unit-time instruction or a call to some data structure $M$, called an $M$-call, that blocks until the answer is returned, and each edge represents a dependency due to the parallel programming primitives. Let $T_{1}$ be the total number of nodes in $D$, and $T_{\infty}$ be the number of nodes on the longest path in $D$. Both designs take work nearly proportional to the Working-Set Bound $W_{L}$ for some legal linearization $L$ of $D$, while having good parallelism. (We assume that each key comparison takes $O(1)$ steps.) The first design, called $M_{1}$, is a simpler batched data structure.

Theorem 3 ( $M_{1}$ Performance). If $P$ uses only $M_{1}$ (i.e., no other data structures), then its running time on $p$ processes using any greedy scheduler is

$$
O\left(\frac{T_{1}+W_{L}+e_{L} \cdot \log p}{p}+T_{\infty}+d \cdot\left((\log p)^{2}+\log n\right)\right)
$$

(as $n, p \rightarrow \infty$ ) for some linearization $L$ of $D$, where $d$ is the maximum number of $M_{1}$-calls on any path in $D$, and $n$ is the maximum size of the map, and $e_{L}$ is the number of small-ops, defined as operations in $L$ that are performed on the map when its size is less than $p$.

Notice that if $M_{1}$ is replaced by an ideal concurrent working-set map (one that does the same work as the sequential working-set map if we ran the program according to linearization $L$ ), then running $P$ on $p$ processors according to the linearization $L$ takes $\Omega\left(T_{\text {opt }}\right)$ worstcase time where $T_{o p t}=\frac{T_{1}+W_{L}}{p}+T_{\infty}$. Also, we very likely have $e_{L} \ll$ $\frac{W_{L}}{\log p}$ in practice, and so can usually ignore the $e_{L} \cdot \log p$ term. Thus $M_{1}$ gives an essentially optimal time bound except for the "span term" $d \cdot\left((\log p)^{2}+\log n\right)$, which adds $O\left((\log p)^{2}+\log n\right)$ time per $M_{1}$-call along some path in $D$. In short, the parallelism of $M_{1}$ is within a factor of $O\left((\log p)^{2}+\log n\right)$ of the optimal.

The second design, called $M_{2}$, uses a more complex pipelined data structure design as well as a weak-priority scheduler (see Section 7.2) to provide a better bound on the "span term".

Theorem 4 ( $M_{2}$ Performance). If $P$ uses only $M_{2}$, then its running time on $p$ processes using any weak-priority scheduler is

$$
O\left(\frac{T_{1}+W_{L}+e_{L} \cdot \log p}{p}+T_{\infty}+d \cdot(\log p)^{2}+s_{L}\right)
$$

for some linearization $L$ of $D$, where $d, e_{L}$ are defined as in Theorem 3, and $s_{L}$ is the weighted span of $D$ where each map operation is weighted by its cost according to $W_{L}$. Specifically, each map operation in $L$ with access rank $r$ is given the weight $\log r+1$, and $s_{L}$ is the maximum weight of any path in $D$. 
Compared to $M_{1}$, the "work term" $\frac{T_{1}+W_{L}+e_{L} \cdot \log p}{p}$ is unchanged, but the "span term" for $M_{2}$ has no $\log n$ term. Since running $P$ on $p$ processors according to the linearization $L$ takes $\Omega\left(T_{o p t}+s_{L}\right)$ worstcase time, $M_{2}$ gives an essentially optimal time bound up to an extra $O\left((\log p)^{2}\right)$ time per map operation along some path in $D$, and hence $M_{2}$ has parallelism within an $O\left((\log p)^{2}\right)$ factor of optimal.

\section{Central Ideas}

We shall now sketch the intuitive motivations behind $M_{1}$ and $M_{2}$.

It starts with Iacono's sequential working-set structure, which contains a sequence of balanced binary search trees $t_{1}, t_{2}, \ldots, t_{l}$ where tree $t_{i}$ for $i<l$ contains $2^{2^{i}}$ items and hence has height $\Theta\left(2^{i}\right)$. The invariant maintained is that the $r$ most recently accessed items are in the first $\log \log r$ trees. A search on a key proceeds by searching in each tree in the sequence in order until the key is found in tree $t_{k}$. After a search, the item is moved to $t_{1}$ and then for each $i<k$, the least recently accessed item from tree $t_{i}$ is moved to tree $t_{i+1}$. By the invariant, any item in the map with recency $r$ will take $O(\log r+1)$ time to access. Each insertion or deletion can be easily carried out in $O(\log (n+1)+1)$ time while preserving the invariant.

The challenge is to 'parallelize' this working-set structure while preserving the total work. The first step is to process operations in batches, using a batched search structure in place of each 'tree'.

The problem is that, if there are $b$ searches for the same item in the last tree, then according to the working-set bound these $b$ operations should take $O(\log n+b)$ work. But if these operations all happen in parallel and end up in the same batch, and we execute this batch naively, then each operation will go through the entire structure leading to $\Omega(b \cdot \log n)$ work.

Therefore, in order to get the desired bound, we must combine duplicate accesses in each batch. But naively sorting a batch of $b$ operations takes $\Theta(b \cdot \log b)$ work. To eliminate this as well, $M_{1}$ (Section 6) uses a novel entropy-sorting algorithm, and a careful analysis yields the desired work bound.

Next, we cannot simply apply the generic "implicit batching" transformation in [3] to $M_{1}$, because the Batcher Bound (Theorem 1 in [3]) would give an expected running time of $O\left(\frac{T_{1}+W+N \cdot s}{p}+T_{\infty}+d \cdot s\right)$ for $N$ map operations, where $W$ is the work done by $M_{1}$, and $s$ is the worst-case span of a size- $p$ batch. The problem is that $s$ is $\Omega(\log n)$, because a batch with a search for an item in the last tree has $\operatorname{span} \Omega(\log n)$.

Firstly, this means that the $N \cdot s$ term would be $\Omega(N \cdot \log n)$, and so the Batcher Bound would be no better than for a batched binary search tree. Secondly, the $d \cdot s$ term would be $\Omega(d \cdot \log n)$. $M_{1}$ has the same span term, because if a cheap operation is 'blocked' by the previous batch that has an expensive operation, then the span of the cheap operation could be $\Omega(\log n)$. To reduce this, we improve $M_{1}$ to $M_{2}$ using an intricate pipelining scheme (explained in Section 7) so that a cheap operation is 'blocked' less by the previous batch.

\section{Parallel Computation Model}

In this section, we describe how the parallel program $P$ generates an execution DAG, how we measure the cost of a given execution DAG, and issues related to the chosen memory model.

\section{Execution DAG}

The actual complete execution of $P$ can be captured by the execution DAG $E$ (which may be schedule-dependent), in which each node is a unit-time instruction and the directed edges represent the underlying computation flow (such as constrained by forking/joining of threads and acquiring/releasing of locks). At any point during the execution of $P$, a node in the program/execution DAG is said to be ready if its parent nodes have been executed. An active thread is simply a ready node in $E$, while a suspended thread is a terminal node in $E$. The program DAG $D$ captures the high-level execution of $P$, but interaction between data structure calls is only captured by the execution DAG. We further assume that all the data structures are (implicitly) batched data structures, and that the number of data structures in use is bounded by some constant. To support implicit batching, each data structure call is automatically handled by a parallel buffer for the data structure. (See Appendix Section A.1.) The execution DAG $E$ consists of core nodes and ds nodes, which are dynamically generated as follows. At the start $E$ has a single core node, corresponding to the start of the program $P$. Each node could be a local instruction or a synchronization instruction (including fork/join and acquire/release of a lock). Each core node could also be a data structure call. When a node is executed, it may generate child nodes or terminate. A join instruction also generates edges that linearize all the join operations according to the actual execution. Likewise, simultaneous operations on a non-blocking lock generate child nodes that are linearized by edges. For a blocking lock, a release instruction generates a child node that is simply the resumed thread that next acquires the lock (if any), with an edge to it from the node corresponding to the originally suspended thread.

The core nodes are further classified into program nodes and buffer nodes. The program nodes (here $P$-nodes) correspond to nodes in the program DAG $D$, and they generate only program nodes except for data structure calls. An $M$-call generates a buffer node corresponding to passing the call to the parallel buffer. This buffer node generates more buffer nodes, until at some point it generates an $M$-node (every $M$-node is a ds node), corresponding to the actual operation on $M$, which passes the input batch to $M$. That $M$-node generates only $M$-nodes except for when it returns the result of some operation in the batch (generating a program node with an edge to it from the original $M$-call), or when it becomes ready for input (generating a buffer node that initiates flushing of the parallel buffer).

\section{Effective Cost}

We shall now precisely define the notion of effective work/span/cost for a parallel data structure used by a (terminating) parallel program. Definition 5 (Effective Work/Span/Cost). Take any program $P$ using a batched data structure $M$ on $p$ processors. Let $E$ be the actual execution DAG of $P$ using $M$. Then the effective work taken by $M$ (as used by $P$ ) is the total number $w$ of $M$-nodes in $E$. And the effective span taken by $M$ is the maximum number $v$ of $M$-nodes on a path in $E$. And the effective cost of $M$ is $\frac{w}{p}+v$.

The effective cost has the desired property that it is subadditive across multiple parallel data structures. This implies that our results are composable with other data structures in this model, since we actually show the following for some linearization $L$ :

^ (Theorem 12 and Theorem 13) $M_{1}$ takes $O\left(W_{L}+e_{L} \cdot \log p\right)$ effective work and $O\left(\frac{W_{L}}{p}+d \cdot\left((\log p)^{2}+\log n\right)\right)$ effective span (using any scheduler). 
^ (Theorem 22 and Theorem 25) $M_{2}$ takes $O\left(W_{L}+e_{L} \cdot \log p\right.$ ) effective work and $O\left(\frac{W_{L}}{p}+d \cdot(\log p)^{2}+s_{L}\right)$ effective span (using a weak-priority scheduler (Section 7.2)).

Interestingly, the bound for the effective cost of $M_{1}$ is independent of the scheduler, while the effective cost bound for $M_{2}$ requires a weak-priority scheduler. In addition, using any greedy scheduler, the parallel buffer for either map $M$ has effective cost (analogously defined) at most $O\left(\frac{T_{1}+w_{M}}{p}+d \cdot \log p\right)$ where $w_{M}$ is the effective work taken by $M$ (Appendix Theorem 26). Therefore our main results (Theorem 3 and Theorem 4 ) follow from the above claims.

\section{Memory Model}

Unless otherwise stated, we work within the pointer machine model for parallel programs given by Goodrich and Kosaraju [26] ${ }^{2}$. But instead of having synchronous processors, we introduce a new more realistic ${ }^{3}$ QRMW model with queued read-modify-write operations (including read, write, test-and-set, fetch-and-add, compare-and-swap) as described in [17], where multiple memory requests to the same memory cell are FIFO-queued and serviced one at a time, and the processor making each memory request is blocked until the request has been serviced. Our data structures can hence be implemented and used in the dynamic multithreading paradigm.

This QRMW pointer machine model supports binary fork/join primitives. It cannot support constant-time random-access locks, but it supports non-blocking locks (try-locks), where attempts to acquire the lock are serialized but do not block. Acquiring a nonblocking lock succeeds if the lock is not currently held but fails otherwise, and releasing always succeeds. If $k$ threads concurrently access a non-blocking lock, then each access completes within $O(k)$ time steps. Non-blocking locks can be used to support activation calls to a process, where activating a process will start its execution iff it is not already executing and it is ready (some condition is satisfied), and the process can optionally reactivate itself on finishing. We can also implement a dedicated lock, which is a blocking lock initialized with keys $[1 . . k]$ for some constant $k$, such that simultaneous acquisitions must be made using distinct keys. When a thread attempts to acquire a dedicated lock, it is guaranteed to obtain the lock after at most $O(1)$ other threads that attempt to acquire the lock at the same time or later.

\section{Amortized Sequential Working-set Map}

In this section we explain the amortized sequential working-set map $M_{0}$, which is similar to Iacono's working-set structure [29], but does not move an accessed item all the way to the front. This localization of self-adjustment is the basis for parallelizing it as in $M_{2}$.

$M_{0}$ keeps the items in a list with segments $S[0 . . l]$. Each segment $S[k]$ has capacity $2^{2^{k}}$ and every segment is full except perhaps the last. Items in each segment are stored in both a key-map and a recency-map, each of which is a BBT (balanced binary tree), sorted by key and by recency respectively. Consider any item $x$ currently in segment $S[k]$. On a search of $x$, if $k=0$ then $x$ is moved to the front (most recent; i.e. first in the recency-map) of $S[0]$, otherwise $x$ is moved to the front of $S[k-1]$ and the last (least recent) item of

\footnotetext{
${ }^{2}$ In short, the main memory can be accessed only via pointers, which can only be stored dereferenced or tested for equality (no pointer arithmetic).

${ }^{3}$ Exclusive reads/writes (EREW) is too strict, while concurrent reads/writes (CRCW) does not realistically model the cost of contention, as stated in [25].
}

$S[k-1]$ is shifted to the front of $S[k]$. On a deletion of $x$, it is removed and for each $i \in[k . . l-1]$ the first (most recent) item of $S[i+1]$ is moved to the back of $S[i]$. On an insertion, the item is added at the back of $S[l]$ (if $S[l]$ is full, then it is added to a new segment $S[l+1]$ ). We now prove an abstract lemma about a list with operations and costs that mimic $M_{0}$. We will later use this same lemma to analyze $M_{1}$ and $M_{2}$ as well.

Lemma 6 (Working-Set Cost Lemma). Take any sequence $L$ of operations on an abstract list $R$, each of which is a search, insert, delete or demote (described below), and a constant $d \geq 0$, such that the following hold (where $n$ is the current size of $R$ ):

$\diamond$ Searching for an item with rank $q$ in $R$ costs $O(\log q+1)$ and it is pulled forward to within the first $2^{d} \cdot q^{1 / 2}$ items in $R$.

今 Searching for an item not in $R$ costs $O(\log (n+1)+1)$.

$\diamond$ Inserting or deleting an item costs $O(\log (n+1)+1)$.

$\diamond$ Demoting an item in $R$ costs 0 and pushes it backward in $R$, but that item subsequently can only be demoted or deleted.

Then the total cost of performing $L$ on $R$ is $O\left(W_{L}\right)$, where demotions are ignored in computing $W_{L}$ (they are not counted as accesses).

Proof. We shall perform the analysis via the accounting method; each operation on $L$ has a budget according to $W_{L}$, and we must use those credits to pay for that operation, possibly saving surplus credits for later. Define the $R$-recency of an item $x$ in $R$ to be the number of items in $R$ that have been inserted before or pulled forward past $x$ in $R$ since the last operation on $x$. Clearly, for each search/insertion/deletion of an item $x$ in $L$, its access rank (actual recency) is at least the $R$-recency of $x$. Each item in $R$ has some stored credit, and we shall maintain the invariant that every item in $R$ with $R$-recency $r$ and stored credit $c$ is within the first $2^{c+2 d+1}+r$ items in $R$ or has been demoted. The invariant trivially holds at the start.

First we show that, on every operation on an item $x$, the invariant can be preserved for $x$ itself. For insertion/deletion or unsuccessful search for $x$, the budget of $\Theta(\log (n+1)+1)$ can pay for the operation and (for insertion) also pay for the stored credit for $x$. For successful search for $x$, it is as follows. Let $c$ be the stored credit and $r$ be the $R$-recency of $x$ before the operation, and let $q$ be the rank of $x$ in $R$ after that. By the invariant, $x$ was within the first $2^{c+2 d+1}+r$ items in $R$ before the operation. Also the budget is $\Omega(\log r+1)$. If $r \geq$ $2^{c+2 d+1}$, then $q \leq 2^{d} \cdot \sqrt{2 r}$ and so the budget can pay for both the operation cost and a new stored credit of $\log \sqrt{2 r}$. If $r \leq 2^{c+2 d+1}$, then $q \leq 2^{d} \cdot \sqrt{2^{c+2 d+1} \cdot 2}=2^{c / 2+2 d+1}$ and so the stored credit can pay for the operation cost and a new stored credit of $c / 2$.

Finally we check that the invariant is preserved for every other item $y$ in $M$. For search/insertion of $x$, the rank of $y$ in $R$ changes by the same amount as its $R$-recency. For deletion of $x$, if $x$ is after $y$ in $R$ then $y$ is more recent than $x$ and so the $R$-recency of $y$ does not change, and if $x$ is before $y$ in $R$ then the rank of $y$ in $R$ decreases by 1 and its $R$-recency decreases by at most 1 . For a demotion, every other item's rank in $R$ does not increase.

This lemma implies that $M_{0}$ has the desired working-set property.

Theorem 7 ( $M_{0}$ Performance). The cost of $M_{0}$ satisfies the working-set bound.

Proof. Let $n$ be the number of items in $M_{0}$. By construction, $2^{l-1} \leq$ $\log (n+1)$, and each operation on $M_{0}$ takes $O\left(2^{k}\right)$ time on segment $S[k]$. Thus each insertion/deletion takes $O(\log (n+1)+1)$ time, and each access/update of an item with rank $q$ in $M_{0}$ (in order of segment 
followed by order in the recency-map) takes $O(\log q+1)$ time. Also, on each access of an item $x$ with rank $q$ in $M_{0}$, its new rank $q^{\prime}$ is at most $2 q^{1 / 2}$, because if $x$ is in $S[0 . .1]$ then $q^{\prime}=1$, and if $x$ is in $S[k]$ for some $k>1$ then $q \geq 2^{2^{k-1}}$ and $q^{\prime} \leq 2^{2^{k-2}} \cdot 2$. Thus by the Working-Set Cost Lemma (Lemma 6) we are done.

\section{Simple Parallel Working-Set Map}

We now present our simple batched working-set map $M_{1}$. The idea is to use the amortized sequential working-set map $M_{0}$ (Section 5) and execute operations in batches ${ }^{4}$. In order to get the bound we desire, however, we must combine operations in a batch that access the same item. In particular, for consecutive accesses to the same item, all but the first one should cost $O(1)$. Therefore, we must sort the batch (efficiently) using the Parallel Entropy Sort (Appendix Definition 29), to 'combine duplicates'. We also control the size of batches - if batches are too small, then we lose parallelism; if the batches are too large, then the sorting cost is too large.

\subsection{Description of $M_{1}$}

As described in the Section $4, M_{1}$-calls in the program are put into the parallel buffer for $M_{1}$. When $M_{1}$ is ready (i.e. the previous batch is done), we flush the parallel buffer to obtain the next input batch $I$, which we cut and store in a feed buffer, which is a queue of bunches (a bunch is a set supporting $O(1)$ addition of a batch and $O(\log b)$-span conversion to a batch if it has size $b)^{5}$ each of size $p^{2}$ except possibly the last. Specifically, we divide $I$ into small batches of size $p^{2}$ except possibly the first and last, the first having size $\min \left(b, p^{2}-q\right)$, where $b$ is the size of $I$ and $q$ is the size of the last bunch $F$ in the feed buffer. Next we add that first small batch to $F$, and append the rest as bunches to the feed buffer. Let $n$ be the current size of $M_{1}$. Then we remove the first $\left\lceil\frac{\log n}{p}\right\rceil$ bunches from the feed buffer, convert them into batches, merge them in parallel into a cut batch $B$, and process it as follows.

First we sort $B$ using the Parallel Entropy Sort (Appendix Definition 29). Then we combine all operations on each item into one group-operation ${ }^{6}$ that is treated as a single operation with the same effect as the whole group of operations in the given order.

We pass the resulting batch (of group-operations) through each segment from $S[0]$ to $S[l]$. At segment $S[k]$, first we search for the relevant items. For insertions, if the item is found then we treat it as an update. For successful searches/updates, we return the results immediately, and shift the items (keeping their relative order) to the front of the previous segment $S[k-1](S[0]$ if $k=0)$. For deletions, if the item is found then we delete the item. Next, we restore the capacity invariant for $S[0 . . k-1]$ — for each segment $S[i]$ from $S[k]$ to $S[1]$, we transfer the appropriate number of items between the front of $S[i]$ and the back of $S[i-1]$ so that either $S[0 . . i-1]$ have total size $\sum_{j=0}^{i-1} 2^{2^{j}}$ or $S[i]$ is empty. Then we pass the unfinished (unreturned) operations (including all deletions) on to the next segment.

At the end, we handle the remaining insertions. First we insert at the back of $S[l]$ up to its capacity. If there are leftover items, we create segments $S\left[l+1 . . l^{\prime}\right]$ with just enough capacity, and carve out the correct amount for each segment from $S\left[l^{\prime}\right]$ to $S[l+1]$ in that order.

\footnotetext{
${ }^{4}$ Each batch is stored in a leaf-based balanced binary tree for efficient processing.

${ }^{5} \mathrm{~A}$ bunch is implemented using a complete binary tree with batches at the leaves, with a linked list threaded through each level to support adding a leaf in $O(1)$ steps.

${ }^{6}$ Each group-operation stores the original operations as a batch.
}

Finally, we return the results for the insertions/deletions and the unsuccessful searches/updates, and we are done processing the batch.

To parallelize the above, we need to be able to efficiently delete, from each segment, any sorted batch of items or any number of the most/least recent items. For this we replace each BBT by a Batched Parallel 2-3 Tree (Appendix Section A.2), where each leaf in the key-map also has a direct pointer to the corresponding leaf in the recency-map, and vice versa. Given a sorted batch of items, we can find them by one batch operation on the key-map, and then we have a batch of direct pointers to the leaves for these items in the recencymap, and hence can perform one reverse-indexing operation on the recency-map to obtain a sorted batch of indices for those leaves, with which we can perform one batch operation to delete them. Similarly, to remove the $b$ most recent items from a segment, we find them via the recency-map, and then do a reverse-indexing operation on the key-map to obtain them in sorted order, whence we can delete them.

\subsection{Analysis of $M_{1}$}

We first bound the cost of parallel entropy-sorting each batch (Lemma 10). To do so, we will find a batch-preserving linearization (Definition 8) $L$ such that for each batch of size $b$, the entropy bound is at most the insert working-set bound (Definition 9), which we in turn bound by the cost according to the working-set bound $W_{L}$, plus $O(\log b)$ per operation when the map is small (i.e. has size $\left.b^{O(1)}\right)$. This extra cost arises when a batch has many operations on distinct items while the map is small, which according to $W_{L}$ are cheap.

Definition 8 (Batch-Preserving Linearization). Take any sequence $I$ of batches of operations on a map $M$. We say that $L$ is a batch-preserving linearization of $I$ if $L$ is a permutation of the operations in $I$ that preserves the ordering of batches and (within each batch) the ordering of operations on the same item.

Definition 9 (Insert Working-Set Bound). The insert workingset bound $I W_{L}$ for any sequence $L$ of map operations is the workingset bound for 'inserting' the items in $L$ in the given order (ignoring the actual operations) into an empty map, namely for each item first searching for it and then inserting it iff it is absent.

Lemma 10 (Batch-Sorting Cost Lemma). Take any sequence $I$ of batches of operations on a map $M$, and any constant $\varepsilon>0$. Then there is a batch-preserving linearization $L$ of $I$ such that parallel entropy-sorting (Appendix Definition 29) each batch in $I$ takes $O\left(W_{L}+\sum_{B \in L} e_{B} \cdot \log b_{B}\right)$ total work over all batches, where each batch $B$ in $L$ has size $b_{B}$ and has $e_{B}$ operations that are performed when $M$ has size less than $b_{B}{ }^{\varepsilon}$ (when $L$ is performed on $M$ ).

Proof. Let $L$ be a batch-preserving linearization of $I$ such that each batch $B$ in $L$ has the maximum insert working-set bound $I W_{B}$ (Definition 9). By the Worse-case Working-set Bound (Appendix Theorem 28) $I W_{B}$ is at least the entropy bound for $B$. Thus parallel entropy-sorting $B$ takes $O\left(I W_{B}\right)$ work (Appendix Theorem 30).

Let $b$ be the size of $B$, and $u$ be the number of distinct items (accessed by operations) in $B$. Partition $B$ into subsequences $B_{0}$ and $B_{1}$ such that $B_{0}$ has only the first operation of every distinct item in $B$. For each $i \in\{0,1\}$, let $C_{i}$ be the cost of the operations in $B_{i}$ according to $W_{L}$, and let $I C_{i}$ be the cost of the operations in $B_{i}$ according to $I W_{B}$, so $I W_{B}=I C_{0}+I C_{1}$. Let $e_{B}$ be the number of operations in $B$ performed when $M$ has size less than $b^{\varepsilon}$ (according to $L$ ).

Note that $I C_{1} \leq C_{1}$ because every operation in $B_{1}$ is a successful search according to $I W_{B}$ with access rank no more than according to $W_{L}$. Thus it suffices to show that $I C_{0} \in O\left(C_{0}+e_{B} \cdot \log b\right)$. 


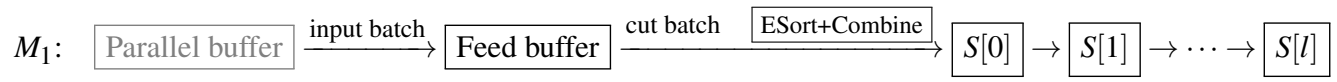

Figure 1: $M_{1}$ Outline

If $e_{B}>\frac{1}{2} u$, then obviously $I C_{0} \in O\left(e_{B} \cdot \log b\right)$.

If $e_{B} \leq \frac{1}{2} u$, then at least $\frac{1}{2} u$ operations in $B_{0}$ are performed when $M$ has size at least $b^{\varepsilon}$. So according to $W_{L}$, each of those operations has access rank at least $b^{\varepsilon}$ and hence costs $\log \left(b^{\varepsilon}\right) \in \Omega(\log u)$. Thus $C_{0} \in \Omega(u \cdot \log u)$. Also, $I C_{0} \in O(u \cdot \log u)$, since any insertion on a map with at most $u$ items has access rank $O(u)$.

Next we prove a simple lemma that allows us to divide the work done on the segments among the operations.

Lemma $11\left(M_{1}\right.$ Segment Work). Each segment $S[k]$ takes $O\left(2^{k}\right)$ work per operation that reaches it.

Proof. Searching/deleting/shifting the relevant items in the parallel 2-3 trees takes $O\left(2^{k}\right)$ work per operation. Also, for each $i \leq k$, the number of transfers (to restore the capacity invariant) between $S[i-1]$ and $S[i]$ is at most the number of operations, and each transfer takes $O\left(2^{i}\right)$ work because there are always at most $\sum_{j=0}^{i} 2^{2^{j}} \leq 2 \cdot 2^{2^{i}}$ items in $S[0 . . i]$. Thus the transfers take $O\left(2^{k}\right)$ total work.

Then we can prove the desired effective work bound for $M_{1}$.

Theorem $12\left(M_{1}\right.$ Effective Work). $M_{1}$ takes $O\left(W_{L}+e_{L} \cdot \log p\right)$ effective work for some linearization $L$ of $D$.

Proof. Cutting the input batch of size $b$ from the parallel buffer into small batches takes $O(b)$ work. Adding the first small batch to the last bunch in the feed buffer takes $O(1)$ work. Inserting the bunches into the feed buffer takes $O(b)$ work. Forming a cut batch of size $b^{\prime}$ (converting the bunches and merging the results) takes $O\left(b^{\prime}\right)$ work. So all this buffering work adds up to $O(1)$ per map operation.

Sorting the (cut) batches takes $O\left(W_{L}+e_{L} \cdot \log p\right)$ total work (over all batches) for some linearization $L$, by the Batch-Sorting Cost Lemma (Lemma 10). Specifically, we choose $\varepsilon=\frac{1}{3}$. For each batch $B$, let $n$ be the size of $M_{1}$ just before that batch, and then $B$ has size $b^{*} \leq$ $\left\lceil\frac{\log n}{p}\right\rceil \cdot p^{2} \leq p \cdot \log n+p^{2}$ and:

$\diamond$ If $n \leq 3 p^{2}$, then $b^{*} \leq p \cdot \log n+p^{2} \leq p \cdot \log \left(3 p^{2}\right)+p^{2} \leq 4 p^{2}$ and so $b^{* \varepsilon} \leq p($ as $p \geq 4)$.

$\diamond$ If $n>3 p^{2}$, then $n>p \cdot \log n+p^{2}+p \geq b^{*}+p$ so none of the operations in that batch can be small-ops.

It now suffices to show that the work on segments is $O\left(W_{L^{\prime}}\right)$ for some linearization $L^{\prime}$ (since either $L$ or $L^{\prime}$ suffices for the final bound). For this, we pretend that a deleted item is marked rather than removed, and when a segment is filled to capacity all marked items are simultaneously transferred to the next segment, and at the last segment the marked items are removed. This takes more work than what $M_{1}$ actually does, but is easier to bound.

We shall now use the Working-Set Cost Lemma (Lemma 6) on the list $R$ of the items in $M_{1}$ (including the marked items) in order of segment followed by recency within the segment, where $R$ is updated after the batch has passed through each segment in the actual execution $A$ of $M_{1}$, and after we finish processing the batch.
We simulate the updates to $R$ by list operations as follows:

$\diamond$ Shift successfully searched/updated items in A: Search for them in reverse order (from back to front in $R$ ).

$\diamond$ Shift marked (to-be-deleted) items in $A$ : Demote them.

$\diamond$ Insert items in $A$ : Insert in the desired positions.

$\diamond$ Remove marked items in $A$ : Delete them.

This simulation yields a sequence $G$ of list operations on $R$, to which we can then apply the Working-Set Cost Lemma.

For each search for an item $x$ with rank $q$ in $R, x$ is found in $S[0]$ or some segment $S[k+1]$ such that $2^{2^{k}}<q$, and so by $M_{1}$ Segment Work (Lemma 11) the search takes $O(\log q+1)$ work in $A$, after which $x$ has new rank in $R$ at most $2 q^{1 / 2}$, like in $M_{0}$ (Theorem 7). After each batch $B$, let $n^{\prime}$ be the final size of $M_{1}$ and $S\left[l^{\prime}\right]$ be the new last segment, and then each insertion in $B$ takes $O\left(\sum_{i=0}^{l^{\prime}} 2^{i}\right) \subseteq$ $O\left(\log n^{\prime}+1\right)$ work in $A$. Each deletion takes $O(\log n+1)$ work in $A$.

Thus by Lemma $6, M_{1}$ takes $O\left(W_{G}\right)$ work on segments. Now let $L^{\prime}$ be the same as $G$ but with each group-operation expanded to its original sequence of operations. Clearly $W_{G} \leq W_{L^{\prime}}$, since each group-operation is on the same item, so we are done.

And now we turn to bounding the effective span.

Theorem $13 \quad\left(M_{1} \quad\right.$ Effective $\quad$ Span). $\quad M_{1}$ takes $O\left(\frac{N}{p}+d \cdot\left((\log p)^{2}+\log n\right)\right)$ effective span, where $N$ is the number of operations on $M_{1}$, and $n$ is the maximum size of $M_{1}$.

Proof. First we bound the the span of processing each cut batch (i.e. the span of the corresponding execution subDAG). Let $s(b)$ denote the maximum span of processing a cut batch of size $b$. Take any cut batch $B$ of size $b$ and let $n_{B}$ be the size of $M_{1}$ just before $B$. $B$ takes $O\left(\frac{b}{p^{2}}+\log b\right)$ span to be removed and formed from the feed buffer, and $O\left((\log b)^{2}\right)$ span to be sorted. $B$ then takes $O\left(\log b+2^{k}\right)$ span in each segment $S[k]$ (because shifting between parallel 2-3 trees of size $O\left(2^{h}\right)$ or cutting a batch of size $O\left(2^{h}\right)$ takes $O(h)$ span), which adds up to $O\left(\log b \cdot \log \log b+\log n_{B}\right)$ span over all segments, since $\log b<2^{k}$ when $k>\log \log b$. Returning the results for each group-operation takes $O(\log b)$ span. Thus $s(b) \in O\left(\frac{b}{p}+(\log b)^{2}+\log n_{B}\right)$. If $b \leq p^{2}$ then $s(b) \in$ $O\left(\frac{b}{p}+(\log p)^{2}+\log n_{B}\right)$. If $b \geq p^{2}$ then $(\log b)^{2} \in O\left(\frac{b}{p}\right)$ and hence $s(b) \in O\left(\frac{b}{p}+\log n_{B}\right)$.

Now let $E$ be the actual execution DAG for $P$ using $M_{1}$ (on $p$ processors). Then the effective span of $M_{2}$ is simply the time taken to execute $E$ on an unlimited number of processors when each $M_{1}$ node in $E$ takes unit time while every other node takes zero time, since $E$ captures all relevant behaviour of $P$ using $M_{1}$ including all the dependencies created by the locks. In this execution, we put a counter at each $M_{1}$-call in the program DAG $D$, initialized to zero, and at each step we increment the counter at every pending $M_{1}$-call (i.e., the result is not yet returned). Then the total number of steps is at most the final counter-weighted span of $D$, which we now bound. 
Take any path $C$ in $D$. Consider each $M_{1}$-call $X$ on $C$. We trace the 'journey' of $X$ from the parallel buffer as an operation in an uncut batch $U$ of size $u$ to a cut batch $B$ of size $b$ to the end of $M_{1}$.

Observe that any batch of size $u$ takes $O(\log p+\log u)$ span to be flushed from the parallel buffer, and $O\left(\log u+\frac{u}{p^{2}}\right)$ span to be cut and added/appended to the bunches in the feed buffer, which in total is at most $O\left(\log p+\frac{u}{p}\right)$ span.

So, first of all, $X$ waits for the preceding uncut batch of size $u^{\prime}$ to be processed, taking $O\left(\log p+\frac{u^{\prime}}{p}\right)$ span. Next, $X$ waits for the current cut batch $B^{\prime}$ of size $b^{\prime}$ to be processed, taking $s\left(b^{\prime}\right)$ span. After that, $U$ is processed, taking $O\left(\log p+\frac{u}{p}\right)$ span. Then $X$ waits for intervening cut batches (between $B^{\prime}$ and $B$ ) with $i$ operations in total. Each intervening batch $B^{*}$ has some size $b^{*} \geq \max \left(p^{2}, p \cdot \log n_{B^{*}}\right)$ and hence $s\left(b^{*}\right) \in O\left(\frac{b^{*}}{p}\right)$. Finally, $B$ is processed, taking $s(b)$ span. Thus $X$ takes $O\left(\log p+\frac{u}{p}+\frac{u^{\prime}}{p}+s(b)+s\left(b^{\prime}\right)+\frac{i}{p}\right)$ span in total.

Note that no two $M_{1}$-calls on the path $C$ can wait for the same intervening batch, because the second can be executed only after the first has returned. Thus over all counters at $M_{1}$-calls on $C$, each of $u, u^{\prime}, b, b^{\prime}, i$ will sum up to at most $N$. Therefore the final counterweighted span of $D$ is at most $O\left(\frac{N}{p}+d \cdot\left((\log p)^{2}+\log n\right)\right)$.

\section{Faster Parallel Working-Set Map}

To reduce the effective span of $M_{1}$, we intuitively have to:

$\diamond$ Shift each accessed item near enough to the front, so that accessing it again soon would be cheap.

$\diamond$ Pipeline the batches somehow, so that an expensive access in a batch does not hold up the next batch.

Naive pipelining will not work, because operations on the same item may take too much work. Hence we shall use a filter before the pipelined segments to ensure that operations proceeding through them are on distinct items, namely we pass all operations through the filter and only allow an operation through if there is not already another operation on the same item in the pipeline.

For similar reasons as in $M_{1}$, we must control both the batch size and filter size to achieve enough parallelism, and so we choose the filter capacity to be $\Theta\left(p^{2}\right)$. However, we cannot put the filter before the first segment, because accessing the filter requires $\Omega(\log p)$ work per operation, whereas to meet the working-set bound we need operations with $O(1)$ access rank to cost only $O(1)$ work.

Therefore, we divide the segments into the first slab and the final slab, where the first slab comprises the first $\log \Theta(\log p)$ segments and the final slab contains the rest, and put the filter after the first slab. Only operations that do not finish in the first slab are passed through the filter, and so the filtering cost per operation is bounded by the $\Theta(\log p)$ work already incurred in going through the first slab. Furthermore, we shift accessed items to the front of the final slab, and 'cascade' the excess items only when a later batch passes.

We cannot pipeline the first slab, but since the first slab is essentially a copy of $M_{1}$ but with only $\log O(\log p)$ trees, its non-pipelined span turns out to be bounded by the $O\left((\log p)^{2}\right)$ span of sorting. To allow operation on items in the first slab to finish quickly, we need to allow the first slab to run while the final slab is running, but only when the filter has size at most $p^{2}$, so that the filter size is always $O\left(p^{2}\right)$.
We also use special locking schemes to guarantee that the first slab and the segments in the final slab can process the operations at a consistent pace without interfering with one another. Finally, we shall weakly prioritize the execution of the final slab, to prevent excessive work from being done in the first slab on an item $x$ if there is already an operation on $x$ in the final slab.

\subsection{Description of $M_{2}$}

We shall now give the details for implementing this (see Figure 2), making considerable use of the Batched Parallel 2-3 Tree (Appendix Section A.2). $M_{2}$ has the same segments as in $M_{1}$, where segment $S[k]$ has assigned capacity $2^{2^{k}}$ but may be under-full or over-full. We shall group the first $m=\left\lceil\log \log \left(2 p^{2}\right)\right\rceil+1$ segments into the first slab, and the other segments into the final slab. $M_{2}$ uses a feed buffer (like $M_{1}$; see Section 6.1), which is a queue of bunches 5 each of size $p^{2}$ except possibly the last.

The $M_{2}$ interface is ready iff both the following hold:

$\diamond$ The parallel buffer or feed buffer is non-empty.

^ The filter has size at most $p^{2}$.

When the $M_{2}$ interface is activated (and ready), it does the following (in sequence) on its run (the locks are described later):

(1) Let $q$ be the size of the last bunch $F$ in the feed buffer. Flush the parallel buffer and cut the input batch of size $b$ into small batches of size $p^{2}$ except possibly the first and last, where the first has size $\min \left(b, p^{2}-q\right)$. Add that first small batch to $F$, and append the others as bunches to the feed buffer. Remove the first bunch from the feed buffer and convert it into a batch $B$, which we shall call a cut batch.

(2) Sort $B$ using the Parallel Entropy Sort (Appendix Definition 29), combining operations on the same item ${ }^{6}$, as in $M_{1}$.

(3) Pass $B$ through the first slab, which processes the operations as in $M_{1}$. Successful searches/updates immediately finish, while the rest finish only if there was no final slab. Successful deletions are tagged to indicate success. But just before running $S[m-1]$ (if it exists) to process the remaining batch at that segment, acquire the neighbour-lock shared with $S[m]$ (as shown in Figure 2) and then acquire the front-lock $F L[0]$.

(4) If there was a final slab, then pass the (sorted) batch of unfinished operations through the filter (including successful deletions), insert the filtered batch into the buffer before $S[m]$, and fork (a child thread) to activate $S[m]$.

(5) Release $F L[0]$ and the neighbour-lock shared with $S[m]$.

(6) Reactivate itself.

The filter is used to ensure that at any point all the operations in the final slab are on distinct items. It is implemented using a batched parallel 2-3 tree that stores items, each tagged with a list of operations on that item (in the order they arrive at the filter) and their cumulative effect (as a single equivalent map operation).

When a batch is passed through the filter, each operation on an item in the filter is appended to the list for it (the effect is also updated) and filtered out of the batch, whereas each operation on an item not in the filter is added to the filter and put into the buffer of $S[\mathrm{~m}]$.

The final slab is pipelined in the following way. Between every pair of consecutive segments is a neighbour-lock, which is a dedicated lock (see Section 4 Memory Model) with 1 key for each arrow to it in Figure 2. Since each segment needs to access the filter and 


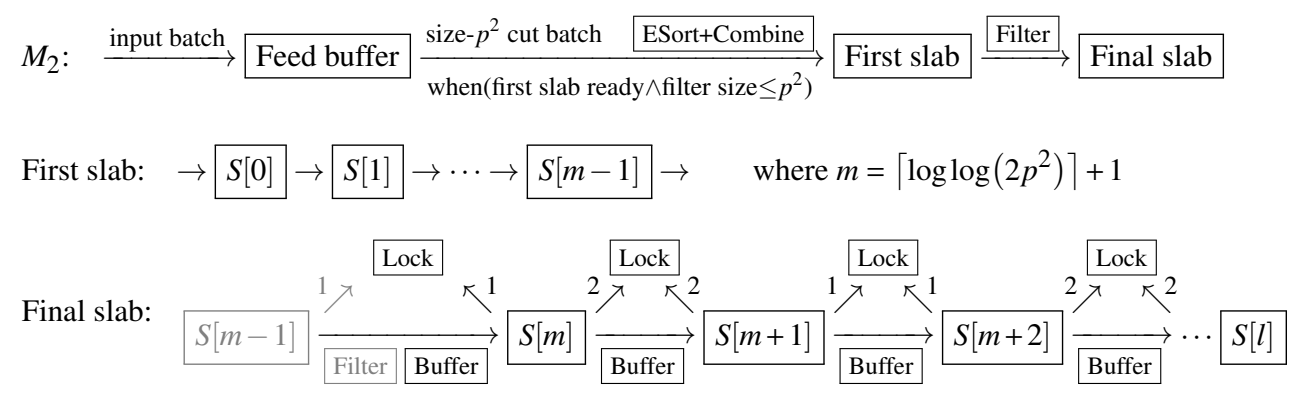

Figure 2: $M_{2}$ Outline

the contents of $S[m]$, those accesses will also be guarded by a frontlocking scheme using a series of front-locks $F L[0 . . l-m]$, each of which is a dedicated lock with 1 key for each arrow to it in Figure 3. (This will be fully spelt out below.)

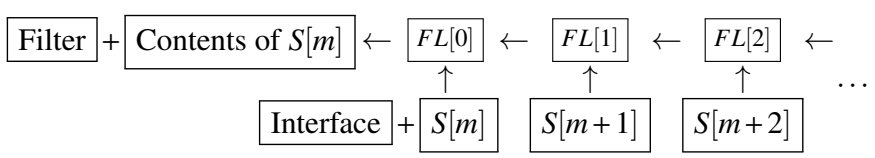

Figure 3: Front-locking

Each final slab segment $S[k]$ has a sorted buffer before it (for operations from $S[k-1]$ ), which is a batched parallel 2-3 tree. $S[k]$ is ready iff its buffer is non-empty, and when activated (and ready) it runs as follows (front-locking is highlighted):

(1) Acquire the neighbour-locks (between $S[k]$ and its neighbours) in the order given by the arrow number in Figure 2.

(2) If $k=m$, acquire $F L[0]$.

(3) If $S[k]$ is the terminal segment and $S[k-1, k]$ have total size exceeding their total capacity, create a new terminal segment $S[k+1]$.

(4) Flush and process the operations in its buffer as follows:

(a) Search for the accessed items $A$ in $S[k]$ (by performing one batch operation on the key-map in $S[k]$ ). Let $R$ be the (sorted batch of) items in $A$ that are found in $S[k]$, and delete $R$ from $S[k]$.

(b) If $k>m$, acquire $F L[k-m]$ to $F L[0]$ in that order.

(c) Search for $R$ in the filter to determine what to do with it. Let $R^{\prime}$ be the items in $R$ to be searched or updated, and delete $R^{\prime}$ from the filter. (Insertions on items in $R$ are treated as updates.) Perform all the updates on items in $R$.

(d) Let $m^{\prime}=\min (k-1, m)$. Fork to return the results for operations on $R^{\prime}$, and insert $R^{\prime}$ at the front of $S\left[m^{\prime}\right]$. If $S[k]$ is (now) the terminal segment, perform all insertions at the front of $S\left[m^{\prime}\right]$, and delete $A \backslash R^{\prime}$ from the filter, and fork to return the results for operations on $A \backslash R^{\prime}$.

(e) If the filter size is at most $p^{2}$, fork to activate the interface.

(f) If $k>m$, release $F L[0]$ to $F L[k-m]$ in that order.

(g) If $S[k-1]$ is over-full, transfer items from the back of $S[k-1]$ to the front of $S[k]$ so that $S[k-1]$ is full.

(h) If $S[k-1]$ is under-full by $i$ items and $S[k]$ has $c$ items and $A$ has $d$ successful deletions, transfer $\min (i, c, d)$ items from the front of $S[k]$ to the back of $S[k-1]$.

(i) If $S[k]$ is not the terminal segment, insert the operations on $A \backslash R^{\prime}$ (with successful deletions tagged as such) into the buffer of $S[k+1]$, then fork to activate $S[k+1]$.
(5) If $S[k]$ is the terminal segment and is empty, remove $S[k]$ to make $S[k-1]$ the new terminal segment.

(6) If $k=m$, release $F L[0]$.

(7) Release both neighbour-locks and reactivate itself.

\subsection{Weak-priority scheduler}

It turns out that, ignoring the sorting cost, all we need to achieve the working-set bound is that, for each operation on an item $x$ in the final slab, the work 'done' by the first slab on $x$ can be counted against the work done in the final slab. This can be ensured using a weakpriority scheduler. A weak-priority scheduler has two queues $Q_{1}$ and $Q_{2}$, where $Q_{1}$ is the high-priority queue, and each ready node is assigned to either $Q_{1}$ or $Q_{2}$, and at every step the following hold:

$\diamond$ If there are $k$ ready nodes, then $\min \left(k, \frac{1}{2} p\right)$ of them are executed.

$\diamond$ If queue $Q_{1}$ has $k$ ready nodes, then $\min \left(k, \frac{1}{2} p\right)$ of them are executed (and so $Q_{1}$ nodes are weakly-prioritized).

The $M_{2}$-nodes generated for the final slab are assigned to $Q_{1}$, while all other $M_{2}$-nodes are assigned to $Q_{2}$. Specifically, each (forked) activation call to $S[\mathrm{~m}]$ and all nodes generated by that are assigned to $Q_{1}$, except for activation calls to the $M_{2}$ interface (which are assigned to $Q_{2}$ ). Any suspended thread is put back into its original queue when it is resumed (i.e. the resuming node is assigned to the same queue as the acquiring node).

\subsection{Analysis of $M_{2}$}

For each computation (subDAG of the actual execution DAG), we shall define its delay, which intuitively captures the minimum possible time it needs, including all waiting on locks. Each blocked acquire of a dedicated lock corresponds to an acquire-stall node $\alpha$ in the execution DAG whose child node $\rho$ is created by the release just before the successful acquisition of the lock. Let $\Delta(\alpha)$ be the ancestor nodes of $\rho$ that have not yet executed at the point when $\alpha$ is executed. Then we define delay as follows.

Definition 14 (Computation Delay). The delay of a computation $\Gamma$ is recursively defined as the weighted span of $\Gamma$, where each acquirestall node $\alpha$ in $\Gamma$ is weighted by the delay of $\Delta(\alpha)$ (to capture the total waiting at $\alpha$ ), and every other node has unit weight.

Also, we classify each operation as segment-bound iff it finishes in some segment, and filter-bound otherwise (namely filtered out due to a prior operation on the same item that finishes in the final slab).

The key lemma (Lemma 19) is that any operation that finishes in segment $S[m+k]$ takes $O\left(2^{m+k}\right)$ delay to be processed by the final slab, which is ensured (Lemma 18) by the front-locking scheme and the balance invariants (Lemma 16). 
Then $M_{2}$ takes $O\left(W_{L}+e_{L} \cdot \log p\right)$ effective work for some linearization $L$ of $D$, and here is the intuitive proof sketch:

(1) The work divides into segment work (done by the slabs) and non-segment work (cutting and sorting batches, and filtering).

(2) The segment work can be divided per operation; segment $S[k]$ does $O\left(2^{k}\right)$ work per operation that reaches it (Lemma 20).

(3) The cutting work is $O(1)$ per operation, and the (overall) sorting work is $O\left(W_{L}+e_{L} \cdot \log p\right)$ for some linearization $L$ of $D$ (Lemma 10). The filtering work is $O(\log p)$ per filtered operation, which can be ignored since each filtered operation already took $\Omega(\log p)$ work in the first slab. Similarly we can ignore the work done in passing the batch through $S[m-1]$.

(4) The segment work on segment-bound operations is $O\left(W_{L^{\prime}}\right)$ for some linearization $L^{\prime}$ of $D$ (Lemma 21; proven using Lemma 6 like we did for $M_{1}$ ).

(5) The segment work on filter-bound operations is $O\left(W_{L^{\prime}}\right)$ too:

(a) We can ignore work during high-busy steps (where $Q_{1}$ has at least $\frac{1}{2} p$ ready nodes), because the final slab takes $O\left(W_{L^{\prime}}\right)$ work and so there are $O\left(\frac{W_{L^{\prime}}}{p}\right)$ high-busy steps.

(b) We can ignore high-busy runs of $S[0 . . m-2]$ (namely with at least half its steps high-busy), because its work is $O(1)$ times the work during high-busy steps.

(c) High-idle runs of $S[0 . . m-2]$ take $O\left(W_{L^{\prime}}\right)$ total work.

(i) Every filter-bound operation is filtered out due to some operation in the final slab. So take any operation $X$ on item $x$ that finishes in a final slab segment $S[k]$.

(ii) During each high-idle run (which takes $\Omega(\log p)$ highidle steps), the processing of $X$ is not blocked by any $Q_{2}$-thread (since $S[0 . . m-2]$ does not hold any neighbour-lock or filter-lock), so each high-idle step 'reduces' its remaining delay, which by the key lemma (Lemma 19) is $O\left(2^{k}\right)$.

(iii) Therefore the work done on $x$ by high-idle runs while $X$ is in the final slab is $O(1)$ times the work done by the final slab on $X$.

Moreover, $M_{2}$ takes $O\left(\frac{W_{L}}{p}+d \cdot(\log p)^{2}+s_{L}\right)$ effective span for some linearization $L$ of $D$ :

(1) The effective span is the time taken to run the execution DAG on infinitely many processors, where each $M_{2}$-node takes unit time while every other node takes zero time.

(2) There are $O\left(\frac{W_{L^{\prime}}}{p}\right)$ filter-full steps (steps in which the filter has size at least $p$ ). To see why, let every operation in the final slab consume a token on each step. Then each filter-full step consumes at least $p$ tokens. But by the key lemma (Lemma 19) the total token consumption is just $O(1)$ times the total work in the final slab, which amounts to $O\left(W_{L^{\prime}}\right)$ (Lemma 21).

(3) There are $O\left(\frac{N}{p}+d \cdot(\log p)^{2}+s_{L^{*}}\right)$ filter-empty steps (filter size at most $p$ ) for some linearization $L^{*}$ of $D$, where $N$ is the number of $M_{2}$-calls, because each operation essentially has the following path: It waits $O\left((\log p)^{2}\right)$ filter-empty steps for the current cut batch in the first slab. Then it waits $O\left(\frac{b^{*}}{p}\right)$ filterempty steps per intervening cut batch of size $b^{*}=p^{2}$. Finally it takes $O\left((\log p)^{2}+\log r\right)$ steps to pass through the slabs where $r$ is its access rank according to $L^{*}$, by the key lemma and the rank invariant (Lemma 24).
We shall now give the details. For the purpose of our analysis, we consider a segment to be running iff it has acquired all neighbourlocks and has not released them. We also consider an operation to be in segment $S[k]$ exactly when it is in the buffer of $S[k]$ or is being processed by $S[k]$ up to step 4 h (inclusive). Likewise, we consider an item that is found in $S[k]$ and to be searched/updated to remain in $S[k]$ until it is shifted to $S\left[\mathrm{~m}^{\prime}\right]$ (in step $4 \mathrm{~d}$ ).

We begin by showing that $M_{2}$ remains 'balanced'; each non-terminal segment has size not too far from capacity.

Definition 15 ( $M_{2}$ Segment Holes). We say that a segment $S[k]$ has $c$ holes if $S[k]$ is not the terminal segment but has $c$ fewer items than its capacity. (If $S[k]$ exceeds capacity then it has no holes.)

Lemma $16\left(M_{2}\right.$ Balance Invariants). The following balance invariants hold:

(1) If $S[m]$ is not running or does not exist, then $S[m-1]$ does not exceed capacity (if it exists).

(2) If the interface is not running, $S[0 . . m-2]$ has no holes, and $S[m-1]$ has at most $d$ holes where $d$ is the number of successful deletions in $S[m]$.

(3) Each final slab segment $S[k]$ has at most $3 \cdot 2^{2^{k}}$ items.

(4) If a final slab segment $S[k]$ is not running, $S[0 . . k-1]$ is at most $2 p^{2}$ below capacity.

Proof. Let $f$ be the filter size. Then $f \leq 2 p^{2}$ always, since the interface only runs if $f \leq p^{2}$, on a batch of size at most $p^{2}$.

$\underline{\text { Invariant } 1}$

$S[m-1]$ can only exceed capacity when $S[m]$ runs, and $S[m]$ restores the invariant (in step $4 \mathrm{~g}$ ) before it finishes running.

Invariant 2

Only the interface creates more holes in $S[0 . . m-1]$ (in step 3), each corresponding to a unique successful deletion that is inserted into the buffer of $S[m]$, and so just after the interface finishes running $S[0 . . m-1]$ has at most $d$ holes where $d$ is the number of successful deletions in $S[m]$, and all the holes must be in $S[m-1]$ since $d \leq$ $2 p^{2} \leq 2^{2^{m-1}}$. Once $S[m]$ runs, $S[m-1]$ will have no holes, because either $S[m]$ was the terminal segment or $S[m]$ had at least $2^{2^{m}}-2 p^{2} \geq$ $d$ items by Invariant 4 .

Invariants 3,4

To establish Invariants 3,4, we can prove sharper invariants. Let $e(k)$ be the total size of $S[0 . . k-1]$ minus their total capacity. Let $u(k)$ be the number of unfinished operations in $S[k+1 . . l]$. Let $d(k)$ be the number of successful deletions in $S[m . . k]$. Then the following invariants hold:

(A) If a final slab segment $S[k]$ is not running, $e(k)+u(k) \leq 2 p^{2}$.

(B) For each final slab segment $S[k]$, we always have $e(k) \leq 4 p^{2}$.

(C) $e(l+1) \leq 2 p^{2}$.

(D) If a final slab segment $S[k]$ is not running, $e(k)+d(k) \geq 0$.

Their proofs can be found at http://arxiv.org/abs/1805.05787.

Using these we can establish Invariants 3,4. By both (B) and (C), for each final slab segment $S[k]$ we have $e(k+1) \leq 4 p^{2}$, and hence $S[k]$ has size at most $\sum_{i=m}^{k} 2^{2^{i}}+4 p^{2} \leq 2 \cdot 2^{2^{k}}+4 p^{2} \leq 3 \cdot 2^{2^{k}}$. By (D), if a final slab segment $S[k]$ is not running, then $-e(k) \leq d(k) \leq f \leq 2 p^{2}$ and hence $S[0 . . k-1]$ is at most $2 p^{2}$ below capacity. 
Corollary 17 ( $M_{2}$ Segment Access Bound). Each batch operation on a parallel 2-3 tree in segment $S[k]$ where $k \geq m-1$ takes $\Theta\left(2^{k}\right)$ work per operation in the batch and $\Theta\left(\log p^{2}+2^{k}\right) \subseteq \Theta\left(2^{k}\right)$ span.

Now we can prove a delay bound on the 'front access' (through the front-locks) by each final slab segment.

Lemma $18\left(M_{2}\right.$ Front Access Bound). Any segment $S[m+k]$ takes $O\left(2^{m+k}\right)$ total delay to acquire the front-locks $F L[0 . . k]$ and run the front-locked section (in-between) and then release $F L[0 . . k]$. And similarly the interface takes $O\left(2^{m}\right)$ delay to acquire $F L[0]$ and run the front-locked section and then release $F L[0]$.

Proof. The front-locked section takes $O\left(2^{m}\right)$ delay, since each operation on a parallel 2-3 tree in $S[m-1, m]$ takes $O\left(2^{m}\right)$ span by Corollary 17 . We shall show by induction that any segment $S[i]$ that has acquired $F L[k]$ will release $F L[k]$ within $c \cdot 2^{m+k}$ delay, where $c$ is a constant chosen to make it true when $k=0$. If $k>0$, then $S[i]$ next attempts to acquire $F L[k-1]$, and if it fails then $S[m+k-1]$ must now be holding it and will release it within $c \cdot 2^{m+k-1}$ delay by induction, and then $S[i]$ will actually acquire $F L[k-1]$ and then will release $F L[k]$ within $c \cdot 2^{m+k-1}$ delay by induction, which in total amounts to $c \cdot 2^{m+k}$ delay. Therefore any segment $S[m+k]$ that attempts to acquire $F L[k]$ will wait at most $c \cdot 2^{m+k}$ delay for any current holder of $F L[k]$ to release it, and then take at most $c \cdot 2^{m+k}$ delay to run its front-locked section and release $F L[0 . . k]$, which is in total $O\left(2^{m+k}\right)$ delay. Similarly for when the interface attempts to acquire $F L[0]$.

Then we can prove the key lemma:

Lemma 19 ( $M_{2}$ Final Slab Bound). Take any segment $S[k]$ where $k \geq m$, and any operation $X$. Then $S[k]$ runs within $O\left(2^{k}\right)$ delay. So if $X$ finishes in segment $S[k]$ then the processing of $X$ in the final slab takes $O\left(2^{k}\right)$ delay.

Proof. Once any $S[k]$ acquires the second neighbour-lock, it will finish within $O\left(2^{k}\right)$ delay, since the operations on the parallel 2-3 trees takes $O\left(2^{k}\right)$ span by Corollary 17, the front access take $O\left(2^{k}\right)$ delay by Lemma 18, and inserting the unfinished operations into the buffer of $S[k+1]$ takes $O(\log p)$ span. Thus once any $S[k]$ acquires the first lock, it waits $O\left(2^{k}\right)$ delay for the holder of the second lock to finish, and then itself finishes within $O\left(2^{k}\right)$ delay. And once the interface acquires the lock shared with $S[m]$, it will finish within $O\left(2^{m}\right)$ delay by Lemma 18 . Thus any $S[k]$ when run will acquire both locks within $O\left(2^{k}\right)$ delay, and then itself finish within $O\left(2^{k}\right)$ delay. Therefore, the final slab takes $O\left(\sum_{i=m}^{k} 2^{i}\right) \subseteq O\left(2^{k}\right)$ delay to process any operation that finishes in $S[k]$.

To bound the total work, we begin by partitioning it per operation:

Lemma 20 ( $M_{2}$ Segment Work). We can divide the segment work (work done on segments) in $M_{2}$ among the operations in the following natural way - each segment $S[k]$ does $\Theta\left(2^{k}\right)$ work per operation it processes.

Proof. If $k<m$, then the proof is the same as for $M_{1}$ Segment Work (Lemma 11). So consider only $k \geq m$. The front-locking takes $O(k) \subseteq$ $O\left(2^{k}\right)$ work, and both accessing the filter and inserting into the buffer of $S[k+1]$ take $O(\log p) \subseteq O\left(2^{k}\right)$ work per operation. Accessing the parallel 2-3 trees takes $\Theta\left(2^{k}\right)$ work per item by Corollary 17 . Thus searching for the accessed items in $S[k]$ takes $O\left(2^{k}\right)$ work per operation, and frontward transfers (in step $4 \mathrm{~h}$ ) can be paid for by the successful deletions.

All that remains is to pay for the rearward transfers (in step $4 \mathrm{~g}$ ). Define the charge for a rearward transfer from $S[k-1]$ to $S[k]$ to be $2^{k-1}$. Let $C$ be the minimum total charge for rearward transfers needed to make every segment not exceed capacity. Then each search/update/insertion that finishes in $S[k]$ increases $C$ by at most the total charge for a cascade of rearward transfers that returns the shifted/inserted item to its original segment, which is less than $2^{k}$. And each rearward transfer done by $S[k]$ is necessary to make $S[k-1]$ not exceed capacity, so it decreases $C$ by at least $2^{k}$. Therefore the searches/updates/insertions can pay for the rearward transfers.

We are now ready to bound the core work done by $M_{2}$ :

Lemma 21 ( $M_{2}$ Core Work). $M_{2}$ takes $O\left(W_{L}\right)$ segment work on segment-bound operations, for some linearization $L$ of $D$.

Proof. The proof is basically the same as for $M_{1}$ (see Theorem 12), and can be found at http://arxiv.org/abs/1805.05787.

We now have the needed lemmas to bound the effective work of $M_{2}$. Theorem $22\left(M_{2}\right.$ Effective Work). $M_{2}$ takes $O\left(W_{L}+e_{L} \cdot \log p\right)$ effective work for some linearization $L$ of $D$.

Proof. We shall follow the same techniques as in the proof of $M_{1}$ Effective Work. We can ignore the work done to transform the input batches from the parallel buffer into cut batches of size $p^{2}$, since it takes $O(1)$ work per operation. Sorting the cut batches takes $O\left(W^{\prime}\right)$ work where $W^{\prime}=W_{L}+e_{L} \cdot \log p$ for some linearization $L$ by BatchSorting Cost Lemma (Lemma 10) with the choice of $\varepsilon=\frac{1}{2}$.

We divide the segment work done among the operations as per Lemma 20. Then the segment-bound operations take $O\left(W_{L^{\prime}}\right)$ total segment work for some linearization $L^{\prime}$ of $D$, by $M_{2}$ Core Work (Lemma 21). We can ignore the work done by $S[m-1]$ and the filter, since that is $O(\log p)$ work per operation, each of which had already taken $\Omega(\log p)$ segment work in $S[0 . . m-2]$. Thus we just have to show that the filter-bound operations take $O\left(W_{L^{\prime}}\right)$ total work in $S[0 . . m-2]$.

We classify each time step as a high-busy step iff $Q_{1}$ has at least $\frac{1}{2} p$ ready nodes, and as a high-idle step otherwise. On each high-busy step, $\frac{1}{2} p$ ready nodes generated for the final slab will be executed (by the weak-priority scheduler). Since the final slab takes $O\left(W_{L^{\prime}}\right)$ work in total by the preceding analysis, there can be only $O\left(\frac{W_{L^{\prime}}}{p}\right)$ high-busy steps, which hence take $O\left(W_{L^{\prime}}\right)$ work.

Now consider each first slab run $\left(M_{2}\right.$ interface step 3$)$ as comprising an $S[0 . . m-2]$ run and then an $S[m-1]$ run (where only the latter is neighbour-locked and front-locked). We classify each $S[0 . . m-2]$ run as a high-busy run iff at least half the time steps during the run are high-busy steps, and as a high-idle run otherwise. During each high-busy run, the total work is $O(1)$ times the work during high-busy steps, since every high-busy step does $\Theta(p)$ work. Thus we can ignore the work done during high-busy runs, and it remains to show that high-idle runs do $O\left(W_{L^{\prime}}\right)$ work on filter-bound operations. Note that every filter-bound operation is trapped in the filter due to some operation that finishes in the final slab. So take any operation $X$ on item $x$ that finishes in segment $S[k]$ in the final slab, and consider the time interval $Z$ when $X$ is in the final slab (after being put in the buffer for $S[m]$ ). Let $\Gamma$ be the remaining computation for the 
processing of $X$, which initially has delay $O\left(2^{k}\right)$ by $M_{2}$ Final Slab Bound (Lemma 19), and note the following:

$\diamond$ At any high-idle step, all ready $Q_{1}$-nodes are executed.

$\diamond$ During any $S[0 . . m-2]$ run, the interface is not holding any neighbour-lock or front-lock.

These imply that during $\Gamma$, at any high-idle step during an $S[0 . . m-2]$ run, the delay of $\Delta(\alpha)$ is reduced for each acquire-stall node $\alpha$ in $\Gamma$ (by structural induction), and hence the delay of $\Gamma$ is reduced. Thus there are $O\left(2^{k}\right)$ such steps during $Z$. Finally observe that each $S[0 . . m-2]$ run takes $\Omega(\log p)$ high-idle steps, and hence at most $O\left(1+\frac{2^{k}}{\log p}\right) S[0 . . m-2]$ runs overlap $Z$, and they do at most $O\left(\log p+2^{k}\right) \subseteq O\left(2^{k}\right)$ work on $x$, which is $O(1)$ times the work done on $X$. Summing over all operations $X$ that finish in the final slab, at most $O\left(W_{L^{\prime}}\right)$ total work is done during $S[0 . . m-2]$ runs on filter-bound operations.

At last, we tackle the effective span bound for $M_{2}$.

Definition 23 ( $M_{2}$ Time Linearization). We say that a linearization $L$ of $D$ is a time linearization if the following hold:

(1) $L$ orders operations by when they finish (for those finished by the final slab this is defined to be when step $4 \mathrm{~d}$ is run).

(2) For operations that finish at the same time in the same segment, $L$ puts searches/updates/insertions in reverse order of how the items are inserted into $S\left[m^{\prime}\right]$ (in step 4d).

Lemma 24 ( $M_{2}$ Rank Invariant). Every item $x$ in the final slab is within the first $r$ items of the final slab, where $r$ is the number of distinct items searched/updated/inserted since the last (combined) operation that shifted/inserted $x$ (in step $4 \mathrm{~d}$ ), according to any time linearization.

Proof. The shifts/inserts can be simulated by individual shifts/inserts of items in the same order as the time linearization, such that every item $x$ that is shifted/inserted is placed at the front of $S\left[m^{\prime}\right]$, at which point the invariant is preserved for $x$. Between consecutive accesses to $x$, every item searched/updated/inserted can move $x$ rearward in $M_{2}$ by at most 1 position.

Theorem $25\left(M_{2}\right.$ Effective Span). $M_{2}$ takes $O\left(\frac{W_{L}}{p}+d \cdot(\log p)^{2}\right.$ $+s_{L}$ ) effective span for some linearization $L$ of $D$.

Proof. See the Section 7.3 outline for the proof sketch. To expand a little on point 3:

Take any path $C$ in the program DAG $D$. Let $L$ be the time linearization of $D$ that puts each $M_{2}$-call along $C$ before all the other operations that finish together with it (in step 4d). Then take any search/update $X$ along $C$ with access rank $r$ according to $L$.

If $X$ finishes in the first slab, it takes $O\left((\log p)^{2}\right)$ steps.

If $X$ finishes in some final slab segment $S[k]$, then just before that $S[k]$ run, the item is within the first $r$ items of the final slab by $M_{2}$ Rank Invariant (Lemma 24). Thus $2^{2^{m}}+r \geq 2^{2^{k-1}}-2 p^{2}$ because $S[0 . . k-1]$ is at most $2 p^{2}$ below capacity by $M_{2}$ Balance Invariants (Lemma 16), and hence by $M_{2}$ Final Slab Bound (Lemma 19) $X$ takes $O\left(2^{k}\right) \subseteq O(\log p+\log r)$ steps to pass through the final slab. Similarly if $X$ finishes in the filter (gets filtered out due to some prior operation $Y$ on the same item that finishes in the final slab).

The full details are at http://arxiv.org/abs/1805.05787.

This concludes our analysis of $M_{2}$.

\section{Practical Schedulers}

The bounds on the effective work and span in Section 6 and Section 7 apply if we use a greedy scheduler for $M_{1}$ and a weak-priority scheduler (Section 7.2) for $M_{2}$. In practice, we do not have such schedulers. But a work-stealing scheduler for $M_{1}$ gives the desired time bound (Theorem 3 ) on average, as essentially shown in $[5,10]$. As for $M_{2}$, dedicating $\frac{1}{2} p$ processors to a greedy scheduler for $Q_{1}$ nodes and the other $\frac{1}{2} p$ processors to another greedy scheduler for $Q_{2}$-nodes gives a weak-priority scheduler as required for $M_{2}$. Replacing each greedy scheduler by a work-stealing scheduler would yield the desired time bound (Theorem 4 ) on average.

\section{Appendix}

Here we spell out the data structures, locking mechanisms and supporting theorems that we have used in our paper. More details and proofs can be found at http://arxiv.org/abs/1805.05787.

\section{A.1 Parallel Buffer}

We can use any parallel buffer implementation that takes $O(p+b)$ work and $O(\log p+\log b)$ span per batch of size $b$, and is such that (regardless of the scheduler) any operation that arrives will be included in the batch that is being flushed or in the next batch, and it always has at most $\frac{1}{2} p+q$ ready nodes (active threads) where $q$ is the number of operations that are currently buffered or being flushed. Then the parallel buffer overhead is given by the following theorem. Theorem 26 (Parallel Buffer Cost). Take any program $P$ using a batched data structure $M$ on $p$ processors using any greedy scheduler. Let $E$ be the actual execution DAG of $P$ using $M$. The buffer's effective cost (see Definition 5) is $O\left(\frac{T_{1}+w_{M}}{p}+d \cdot \log p\right)$, where $w_{M}$ is the effective work taken by $M$ and $d$ is the maximum number of $M$-calls on any path in the program DAG $D$ for $P$.

\section{A.2 Batched Parallel 2-3 Tree}

A batched parallel 2-3 tree $M$ supports the following batched operations (where $n$ is the number of items in $M$ before the batch):

(1) Normal operation on an item-sorted batch $B$ of $b$ operations on distinct items: $M$ performs all the operations in $B$, and returns (as a batch) the results in the same order as in $B$, all within $O(b \cdot \log n)$ work and $O(\log b+\log n)$ span. The result for each operation is a direct pointer to the item in $M$ (or null if deleted).

(2) Reverse-indexing operation on an (unsorted) input batch $B$ of $b$ direct pointers to distinct items in $M: M$ returns the itemsorted batch of the items pointed to by the direct pointers in $B$, all within $O(b \cdot \log n)$ work and $O(\log b+\log n)$ span.

The batched parallel 2-3 tree that we use can be adapted from the parallel 2-3 dictionary described in [34].

\section{A.3 Sorting Theorems}

Let $S$ be the set of items in the search problem, linearly ordered by a given comparison function. We assume that $\#(S)>1$.

Definition 27 (Normalized Frequencies). We say that $q_{1 . . u}$ are normalized frequencies if $q_{1 . . u} \in \mathbb{R}^{+}$and $\sum_{i=1}^{u} q_{i}=1$. Let $\operatorname{Seq}(n, q)$ be the class of sequences in $S^{n}$ with normalized item frequencies $q$. Theorem 28 (Worse-case Working-set Bound). Take any normalized frequencies $q_{1 . . u}$, and let $C=\operatorname{Seq}(n, q)$. Then the working-set bound for inserting some sequence $I \in C$ is $\Omega(n \cdot H+n)$ where $H=\sum_{i=1}^{u}\left(q_{i} \cdot \ln \frac{1}{q_{i}}\right)$. 
Definition 29 (Parallel Entropy Sort). Let PESort be the following parallel variant of Quicksort:

Use the Parallel Pivot Algorithm to pick a pivot from the middle half of the input list. Parallel partition the list into a lower, a middle (equal to the pivot) and an upper part. Then sort the lower and upper parts recursively. Finally concatenate the three parts.

Theorem 30 (PESort Performance). Take any normalized frequencies $q_{1 . . u}$, and let $C=\operatorname{Seq}(n, q)$. Then PESort sorts every sequence from $C$, taking $O(n \cdot H+n)$ work and $O\left((\log n)^{2}\right)$ span, where $H=\sum_{i=1}^{u}\left(q_{i} \cdot \ln \frac{1}{q_{i}}\right)$.

Theorem 31 (Parallel Pivot Algorithm). Let PPivot be the following parallel algorithm:

Partition the input list of size $k$ into blocks $B[1 . . c]$ of size $\log k$ except perhaps the last block. For each $i$ in $[1 . . c]$ in parallel, find the median $m[i]$ of $B[i]$ using the linear-time algorithm. Then use an optimal parallel sorting algorithm (such as adapted from $[6,26])$ to sort $m[1 . . c]$ and output their median.

Then PPivot outputs an item that is in the two middle quartiles of any input list of size $k$, taking $O(k)$ work and $O(\log (k))$ span.

\section{Acknowledgments}

This research was supported in part by Singapore MOE AcRF Tier 1 grant T1 251RES1719 and National Science Foundation grants CCF-1150036, CCF-1733873 and CCF-1725647.

\section{References}

[1] Yehuda Afek, Haim Kaplan, Boris Korenfeld, Adam Morrison, and Robert Endre Tarjan. 2012. CBTree: A Practical Concurrent Self-Adjusting Search Tree. In Distributed Computing - 26th International Symposium, DISC 2012, Salvador, Brazil, October 16-18, 2012. Proceedings. 1-15. https://doi.org/10.1007/ 978-3-642-33651-5 1

[2] Yehuda Afek, Haim Kaplan, Boris Korenfeld, Adam Morrison, and Robert Endre Tarjan. 2014. The CB tree: a practical concurrent self-adjusting search tree. Distributed Computing 27, 6 (2014), 393-417. https://doi.org/10.1007/ s00446-014-0229-0

[3] Kunal Agrawal, Jeremy T Fineman, Kefu Lu, Brendan Sheridan, Jim Sukha, and Robert Utterback. 2014. Provably good scheduling for parallel programs that use data structures through implicit batching. In Proceedings of the 26th ACM symposium on Parallelism in algorithms and architectures. ACM, 84-95.

[4] Yaroslav Akhremtsev and Peter Sanders. 2016. Fast Parallel Operations on Search Trees. In 23rd IEEE International Conference on High Performance Computing, HiPC 2016, Hyderabad, India, December 19-22, 2016. 291-300. https://doi.org/ 10.1109/HiPC.2016.042

[5] Nimar S Arora, Robert D Blumofe, and C Greg Plaxton. 2001. Thread scheduling for multiprogrammed multiprocessors. Theory of computing systems 34,2 (2001), $115-144$.

[6] Mikhail J Atallah, Richard Cole, and Michael T Goodrich. 1989. Cascading divideand-conquer: A technique for designing parallel algorithms. SIAM J. Comput. 18 3 (1989), 499-532.

[7] Guy E. Blelloch, Daniel Ferizovic, and Yihan Sun. 2016. Just Join for Parallel Ordered Sets. In Proceedings of the 28th ACM Symposium on Parallelism in Algorithms and Architectures, SPAA 2016, Asilomar State Beach/Pacific Grove, CA, USA, July 11-13, 2016. 253-264. https://doi.org/10.1145/2935764.2935768

[8] Guy E. Blelloch and Margaret Reid-Miller. 1997. Pipelining with Futures. In Proceedings of the ACM Symposium on Parallel Algorithms and Architectures (SPAA '97). ACM, New York, NY, USA, 249-259. https://doi.org/10.1145/ 258492.258517

[9] Guy E. Blelloch and Margaret Reid-Miller. 1998. Fast Set Operations Using Treaps. In SPAA. 16-26. https://doi.org/10.1145/277651.277660

[10] Robert D Blumofe and Charles E Leiserson. 1999. Scheduling multithreaded computations by work stealing. Journal of the ACM (JACM) 46, 5 (1999), 720 748.

[11] R. P. Brent. 1974. The Parallel Evaluation of General Arithmetic Expressions. J. ACM (1974), 201-206.

[12] Gerth Stølting Brodal, Jesper Larsson Träff, and Christos D. Zaroliagis. 1998. A
Parallel Priority Queue with Constant Time Operations. J. Parallel and Distrib. Comput. (1998), 4-21.

[13] Trevor Brown, Faith Ellen, and Eric Ruppert. 2014. A general technique for non-blocking trees. In ACM SIGPLAN Symposium on Principles and Practice of Parallel Programming, PPoPP '14, Orlando, FL, USA, February 15-19, 2014. 329-342. https://doi.org/10.1145/2555243.2555267

[14] Thomas H. Cormen, Charles E. Leiserson, Ronald L. Rivest, and Clifford Stein. 2009. Introduction to Algorithms (third ed.). The MIT Press.

[15] A. Crauser, K. Mehlhorn, U. Meyer, and P. Sanders. 1998. A Parallelization of Dijkstra's Shortest Path Algorithm. In Proceedings of the International Symposium on Mathematical Foundations of Computer Science (MFCS). Springer, 722-731.

[16] James R. Driscoll, Harold N. Gabow, Ruth Shrairman, and Robert E. Tarjan. 1988. Relaxed heaps: an alternative to Fibonacci heaps with applications to parallel computation. Commun. ACM 31 (1988), 1343-1354. Issue 11.

[17] Cynthia Dwork, Maurice Herlihy, and Orli Waarts. 1997. Contention in shared memory algorithms. Journal of the ACM (JACM) 44, 6 (1997), 779-805.

[18] Faith Ellen, Panagiota Fatourou, Joanna Helga, and Eric Ruppert. 2014. The amortized complexity of non-blocking binary search trees. In ACM Symposium on Principles of Distributed Computing, PODC '14, Paris, France, July 15-18, 2014. 332-340. https://doi.org/10.1145/2611462.2611486

[19] Faith Ellen, Panagiota Fatourou, Eric Ruppert, and Franck van Breugel. 2010. Non-blocking Binary Search Trees. In Proceedings of the 29th ACM SIGACTSIGOPS Symposium on Principles of Distributed Computing (PODC '10). ACM, New York, NY, USA, 131-140. https://doi.org/10.1145/1835698.1835736

[20] Amr Elmasry, Arash Farzan, and John Iacono. 2013. On the hierarchy of distribution-sensitive properties for data structures. Acta informatica 50, 4 (2013), 289-295.

[21] Stephan Erb, Moritz Kobitzsch, and Peter Sanders. 2014. Parallel Bi-Objective Shortest Paths using Weight-Balanced B-Trees with Bulk Updates. In Proceedings of the Symposium on Experimental Algorithms (SEA). to appear

[22] Panagiota Fatourou and Nikolaos D. Kallimanis. 2012. Revisiting the combining synchronization technique. In PPoPP. 257-266. https://doi.org/10.1145/2145816. 2145849

[23] Leonor Frias and Johannes Singler. 2007. Parallelization of Bulk Operations for STL Dictionaries. In Euro-Par Workshops (LNCS), Vol. 4854. Springer, 49-58.

[24] Matteo Frigo, Charles E. Leiserson, and Keith H. Randall. 1998. The Implementation of the Cilk-5 Multithreaded Language. In Proceedings of the ACM SIGPLAN Conference on Programming Language Design and Implementation (PLDI). 212-223.

[25] Phillip B Gibbons, Yossi Matias, and Vijaya Ramachandran. 1998. The QueueRead Queue-Write PRAM model: Accounting for contention in parallel algorithms. SIAM J. Comput. 28, 2 (1998), 733-769.

[26] Michael T Goodrich and S Rao Kosaraju. 1996. Sorting on a parallel pointer machine with applications to set expression evaluation. Journal of the ACM (JACM) 43, 2 (1996), 331-361.

[27] R. L. Graham. 1969. Bounds on Multiprocessing Anomalies. SIAM J. Appl. Math. (1969), 17(2):416-429.

[28] Danny Hendler, Itai Incze, Nir Shavit, and Moran Tzafrir. 2010. Flat combining and the synchronization-parallelism tradeoff. In Proceedings of the ACM Symposium on Parallelism in Algorithms and Architectures (SPAA). 355-364. https://doi.org/10.1145/1810479.1810540

[29] John Iacono. 2001. Alternatives to splay trees with $\mathrm{O}(\log n)$ worst-case access times. In Proceedings of the twelfth annual ACM-SIAM symposium on Discrete algorithms. Society for Industrial and Applied Mathematics, 516-522.

[30] Intel Corporation 2013. Intel Cilk Plus Language Extension Specification, Version 1.1. Intel Corporation. Document 324396-002US. Available from http://cilkplus. $\mathrm{org} / \mathrm{sites} /$ default/files/open_specifications/Intel_Cilk_plus_lang_spec_2.htm.

[31] OpenMP Architecture Review Board. 2013. OpenMP Application Program Interface, Version 4.0. Available from http://www.openmp.org/mp-documents/ OpenMP4.0.0.pdf.

[32] Y. Oyama, K. Taura, and A. Yonezawa. 1999. Executing Parallel Programs With Synchronization Bottlenecks Efficiently. In Proceedings of the International Workshop on Parallel and Distributed Computing for Symbolic and Irregular Applications (PDSIA). 182-204.

[33] Richard C. Paige and Clyde P. Kruskal. 1985. Parallel Algorithms for Shortest Path Problems. In Int. Conference on Parallel Processing. 14-20.

[34] Wolfgang Paul, Uzi Vishkin, and Hubert Wagener. 1983. Parallel dictionaries on 2-3 trees. Automata, Languages and Programming (1983), 597-609.

[35] James Reinders. 2007. Intel Threading Building Blocks: Outfitting C++for Multi-Core Processor Parallelism. O'Reilly.

[36] Peter Sanders. 1998. Randomized Priority Queues for Fast Parallel Access. Journal of Parallel Distributed Computing 49, 1 (1998), 86-97.

[37] Daniel Dominic Sleator and Robert Endre Tarjan. 1985. Self-adjusting binary search trees. Journal of the ACM (JACM) 32, 3 (1985), 652-686.

[38] tpl 2007. The Task Parallel Library. http://msdn.microsoft.com/en-us/magazine/ cc163340.aspx. http://msdn.microsoft.com/en-us/magazine/cc163340.aspx 